\title{
Understanding and Improving Computational Science Storage Access through Continuous Characterization
}

\author{
Philip Carns, ${ }^{*}$ Kevin Harms, ${ }^{\dagger}$ William Allcock, ${ }^{\dagger}$ Charles Bacon, ${ }^{\dagger}$ Samuel Lang, ${ }^{*}$ Robert Latham, ${ }^{*}$ and Robert Ross* \\ ${ }^{*}$ Mathematics and Computer Science Division, ${ }^{\dagger}$ Argonne Leadership Computing Facility \\ Argonne National Laboratory \\ Argonne, IL 60439 \\ * $\{$ carns,slang,robl,robr $\} @$ mcs.anl.gov, ${ }^{\dagger}\{$ harms,allcock,bacon $\} @$ alcf.anl.gov
}

\begin{abstract}
Computational science applications are driving a demand for increasingly powerful storage systems. While many techniques are available for capturing the $I / O$ behavior of individual application trial runs and specific components of the storage system, continuous characterization of a production system remains a daunting challenge for systems with hundreds of thousands of compute cores and multiple petabytes of storage. As a result, these storage systems are often designed without a clear understanding of the diverse computational science workloads they will support.

In this study, we outline a methodology for scalable, continuous, systemwide I/O characterization that combines storage device instrumentation, static file system analysis, and a new mechanism for capturing detailed application-level behavior. This methodology allows us to quantify systemwide trends such as the way application behavior changes with job size, the "burstiness" of the storage system, and the evolution of file system contents over time. The data also can be examined by application domain to determine the most prolific storage users and also investigate how their I/O strategies correlate with $I / O$ performance. At the most detailed level, our characterization methodology can also be used to focus on individual applications and guide tuning efforts for those applications.

We demonstrate the effectiveness of our methodology by performing a multilevel, two-month study of Intrepid, a 557teraflop IBM Blue Gene/P system. During that time, we captured application-level $\mathrm{I} / \mathrm{O}$ characterizations from 6,481 unique jobs spanning 38 science and engineering projects with up to 163,840 processes per job. We also captured patterns of $\mathrm{I} / \mathrm{O}$ activity in over 8 petabytes of block device traffic and summarized the contents of file systems containing over 191 million files. We then used the results of our study to tune example applications, highlight trends that impact the design of future storage systems, and identify opportunities for improvement in $\mathrm{I} / \mathrm{O}$ characterization methodology.
\end{abstract}

\section{INTRODUCTION}

Computational science applications are driving a demand for increasingly powerful storage systems. This situation is true especially on leadership-class systems, such as the 557 TFlop IBM Blue Gene/P at Argonne National Laboratory, where the storage system must meet the concurrent $\mathrm{I} / \mathrm{O}$ requirements of hundreds of thousands of compute elements [1]. Hardware architecture, file systems, and middleware all contribute to providing high-performance I/O capabilities in this environment. These components cannot be considered in isolation, however. The efficiency of the storage system is ultimately determined by the nature of the data stored on it and the way applications choose to access that data. Understanding storage access characteristics of computational science applications is therefore a critical—and challenging — aspect of storage optimization.

Various methods exist for analyzing application access characteristics and their effect on storage. Synthetic I/O benchmarks are easily instrumented and parameterized, but in many cases they fail to accurately reflect the behavior of scientific applications [2], [3], [4]. Application-based benchmarks are more likely to reflect actual production behavior, but the available benchmarks do not represent the variety of scientific domains and applications seen on leadership-class machines, each with unique access characteristics and data requirements. $\mathrm{I} / \mathrm{O}$ tracing at the application, network, or storage level is another technique that has been successful in analyzing generalpurpose network file systems [5], [6]. However, these tracing techniques are impractical for capturing the immense volume of I/O activity on leadership-class computer systems. These systems leverage high-performance networks and thousands of hard disks to satisfy I/O workloads generated by hundreds of thousands of concurrent processes. Instrumentation at the component level would generate an unmanageable quantity of data. Such systems are also highly sensitive to perturbations in performance that may be introduced by comprehensive tracing.

As a result, key gaps exist in our understanding of the storage access characteristics of computational science applications on leadership-class systems. To address this deficiency, we have developed an application-level I/O characterization tool, known as Darshan [7], that captures relevant I/O behavior at production scale with negligible overhead. In this work we deploy Darshan in conjunction with tools for block device monitoring and static file system analysis in order to answer the following questions for a large-scale production system: 
- What applications are running, what interfaces are they using, and who are the biggest I/O producers and consumers?

- How busy is the I/O system, how many files are being created of what size, and how "bursty" is $\mathrm{I} / \mathrm{O}$ ?

- What I/O interfaces and strategies are employed by the top I/O producers and consumers? How successful are they in attaining high $\mathrm{I} / \mathrm{O}$ efficiency? Why?

To answer these questions, we performed a long-running, multilevel I/O study of the Intrepid Blue Gene/P (BG/P) system at Argonne National Laboratory. The study spanned two months of production activity from January to March 2010. During that time we recorded three aspects of $\mathrm{I} / \mathrm{O}$ behavior: storage device activity, file system contents, and application I/O characteristics. We combine these three sources of information to present a comprehensive view of storage access characteristics and their relationships. We also show how this data can be used to identify production applications with $\mathrm{I} / \mathrm{O}$ performance problems as well as guide efforts to improve them.

\section{Summary of contributions}

In Section II we outline a methodology for comprehensive I/O characterization of HPC systems. In Sections III, IV, and $\mathrm{V}$ we demonstrate how this methodology enables continuous analysis of production $\mathrm{I} / \mathrm{O}$ activity at an unprecedented scale. We investigate the I/O characteristics of a 557-teraflop computing facility over a two-month period and uncover a variety of unexpected application I/O trends. In Section VI we demonstrate how this same methodology can be used to tune and improve the I/O efficiency of specific applications. We conclude in Section VIII by identifying the impact of our analysis on future I/O subsystem designs and research directions.

\section{TARGET SYSTEM AND METHODOLOGY}

This study was conducted on Intrepid, the IBM BG/P system at the Argonne Leadership Computing Facility (ALCF). The ALCF makes large allocations available to the computational science community through the Innovative and Novel Computational Impact on Theory and Experiment (INCITE) program [8]. Systems such as Intrepid therefore host a diverse set of applications from scientific domains including climate, physics, combustion, and Earth sciences.

Intrepid is a 163,840 -core production system with $80 \mathrm{TiB}$ of RAM and a peak performance of 557 TFlops. The primary high-performance storage system employs 128 file servers running both PVFS [1] and GPFS [9], with a separate, smaller home directory volume. Data is stored on 16 DataDirect Networks S2A9900 SANs. The storage system has a total capacity of $5.2 \mathrm{PiB}$ and a peak $\mathrm{I} / \mathrm{O}$ rate of approximately $78 \mathrm{GiB} / \mathrm{s}$. The architecture and scalability of this storage system have been analyzed in detail in a previous study [1].

Intrepid groups compute nodes (CNs) into partitions of sizes 512, 1,024, 2,048, 8,192, 16,384, 32,768, and 40,960 nodes. All jobs must select one of these partition sizes, regardless of the number of nodes that will be used. Each set of 64 compute nodes utilizes a single, dedicated I/O forwarding node (ION). This ION provides a single 10 gigabit Ethernet link to storage that is shared by the CNs.

The goal of our characterization methodology on this system was to gain a complete view of I/O behavior by combining data from multiple levels of the I/O infrastructure without disrupting production activity. To accomplish this goal, we captured data in three ways. We monitored storage device activity, periodically analyzed file system contents, and instrumented application-level I/O activity using a tool that we developed for that purpose.

\section{A. Characterizing storage device activity}

On the storage side of the system, the DataDirect Networks SANs used by PVFS and GPFS are divided into sets of LUNs that are presented to each of the 128 file servers. Activity for both file systems was captured by observing traffic at the block device level. We recorded high-level characteristics such as bandwidth, amount of data read and written, percentage of utilization, and average response times.

Behavior was observed by using the iostat command-line tool included with the Sysstat collection of utilities [10]. Iostat can report statistics for each block device on regular intervals. We developed a small set of wrappers (known as iostat-mon) to monitor iostat data on each file server. Data was collected every 60 seconds, logged in a compact format, and then postprocessed to produce aggregate summaries. All local disk activity was filtered out to eliminate noise from operating system activity. The data was collected continuously from January 23 to March 26, but four days of data were lost in February because of an administrative error.

\section{B. Characterizing file system contents}

The block device instrumentation described above captures all data movement, but it does not describe the nature of the persistent data that is retained on the system. To capture this information, we used the fsstats [11] tool. Fsstats analyzes entire directory hierarchies to collect a snapshot of static characteristics, such as file sizes, file ages, capacity, and a variety of namespace attributes. We ran the fsstats tool at the beginning and end of the study on both primary file systems. GPFS was measured on January 23 and March 25, while PVFS was measured on January 27 and March 31. This approach allowed us to observe the change in file system contents over the course of the study.

\section{Characterizing application behavior}

The most detailed level of characterization was performed by analyzing application-level access characteristics. Application-level characterization is critical because it captures I/O access patterns before they are altered by high-level libraries or file systems. It also ensures that system behavior can be correlated with the specific job that triggered it. Application-level I/O characterization has traditionally been a challenge for arbitrary production workloads at scale, however. 


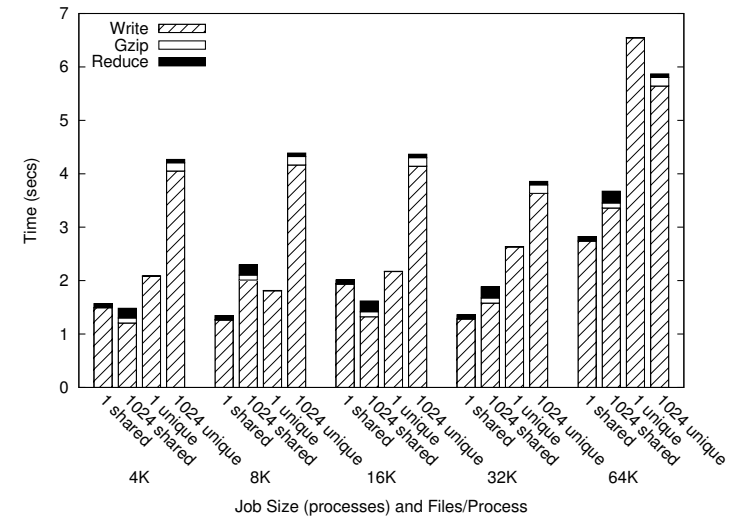

Fig. 1. Darshan output time for varying BG/P job sizes.

Tracing and logging each I/O operation become expensive (in terms of both overhead and storage space) at scale, while approaches that rely on statistical sampling may fail to capture critical behavior. In previous work we developed a tool called Darshan [7] in order to bridge this gap. Darshan captures information about each file opened by the application. Rather than trace all operation parameters, however, Darshan captures key characteristics that can be processed and stored in a compact format. Darshan instruments POSIX, MPI-IO, Parallel netCDF, and HDF5 functions in order to collect a variety of information. Examples include access patterns, access sizes, time spent performing I/O operations, operation counters, alignment, and datatype usage. Note that Darshan performs explicit capture of all I/O functions rather than periodic sampling in order to ensure that all data is accounted for.

The data that Darshan collects is recorded in a bounded (approximately $2 \mathrm{MiB}$ maximum) amount of memory on each MPI process. If this memory is exhausted, then Darshan falls back to recording coarser-grained information, but we have yet to observe this corner case in practice. Darshan performs no communication or $\mathrm{I} / \mathrm{O}$ while the job is executing. This is an important design decision because it ensures that Darshan introduces no additional communication synchronization or I/O delays that would perturb application performance or limit scalability. Darshan delays all communication and I/O activity until the job is shutting down. At that time Darshan performs three steps. First it identifies files that were shared across processes and reduces the data for those files into an aggregate record using scalable MPI collective operations. Each process then compresses the remaining data in parallel using Zlib. The compressed data is written in parallel to a single binary data file. Figure 1 shows the Darshan output time for various job sizes on Intrepid as measured in previous work [7]. This figure shows four cases for each job size: a single shared file, 1,024 shared files, one file per process, and 1,024 files per process. The largest case demonstrates that the Darshan shutdown process can be performed in less than 7 seconds even for jobs with 65,336 processes that opened 67 million files [7]. This time is not likely to be noticeable because jobs at this scale take several minutes to boot and shut down. In addition, we have measured the overhead per file system operation to be less than $0.05 \%$, even for operations that read only a single byte of data.

Darshan was installed on Intrepid through modifications to the default MPI compilers. Users who built MPI applications using these default compilers were therefore automatically included in the study. Darshan did not achieve complete coverage of all applications, however. Some applications were compiled prior to the Darshan deployment on January 14, 2010. Other applications either did not use MPI at all or used custom build scripts that had not been modified to link in Darshan. Users do have the option of explicitly disabling Darshan at compile time or run time, though this option is rarely chosen. In total, Darshan characterized $27 \%$ of all jobs executed during the interval studied in this work.

To analyze the resulting data from Darshan, we postprocessed all log files and loaded the resulting data into a unified SQL database. We also utilized a graphical tool included with Darshan to generate summary reports for particular jobs of interest. This tool is available to users and system administrators; it enables immediate feedback on the I/O behavior of any production job, in many cases eliminating the need to explicitly instrument or rerun jobs in order to troubleshoot I/O performance problems.

\section{Performance metrics}

One important metric for applications is aggregate $I / O$ bandwidth. For parallel I/O benchmarks we typically calculate this by dividing the amount of data moved by the time of the slowest MPI process, with some coordination ensuring that I/O overlapped. In this work we are observing real applications running in production, and these applications may not have the same coordination seen in I/O benchmarks. Additionally, because the applications are running across a range of job sizes, it is useful for comparison purposes to examine performance relative to job size, rather than as an absolute value. We introduce a generic metric for read and write performance that can be derived from the Darshan statistics of unmodified applications and scaled across a variety of job sizes.

Darshan records independent statistics for each file accessed by the application, including the number of bytes moved, cumulative time spent in I/O operations such as read () and write (), and cumulative time spent in metadata operations such as open () and stat (). The aggregate I/O bandwidth can be estimated by dividing the total amount of data transferred by the amount of I/O time consumed in the slowest MPI process. To make comparisons across jobs of different sizes, we divide the aggregate performance by the number of compute nodes allocated to the job. The result is a MiB per second per compute node $(\mathrm{MiB} / \mathrm{s} / \mathrm{CN})$ metric, calculated as follows:

$$
M i B / s / C N=\left(\frac{\sum_{r a n k=0}^{n-1}\left(\text { bytes }_{r}+\text { bytes }_{w}\right)}{\max _{\text {rank }=0}^{n-1}\left(t_{m d}+t_{r}+t_{w}\right)}\right) / N_{c n} .
$$

In this equation, $n$ represents the number of MPI processes, while $N_{c n}$ represents the number of compute nodes. Intrepid 
TABLE I

PERFORMANCE METRICS FOR IOR EXAMPLES

\begin{tabular}{l|r|rr}
\hline & Actual & \multicolumn{2}{|c}{ Estimated } \\
Example & $\mathrm{MiB} / \mathrm{s}$ & $\mathrm{MiB} / \mathrm{s}$ & $\mathrm{MiB} / \mathrm{s} / \mathrm{CN}$ \\
\hline IOR N-1 write & 4021.01 & 4026.91 & 3.93 \\
IOR N-1 read & 6050.73 & 6067.70 & 5.93 \\
IOR N-N write & 3957.88 & 4050.39 & 3.96 \\
IOR N-N read & 5877.41 & 5883.73 & 5.75 \\
\hline
\end{tabular}

has four cores per compute node, so those two numbers seldom match. Here bytes $s_{r}$ and bytes $s_{w}$ represent the number of bytes read and written by the MPI process, respectively, while $t_{m d}$, $t_{r}$, and $t_{w}$ represent time spent in metadata, read, and write operations, respectively. A slight variation is used to account for shared files because, in that scenario, Darshan combines statistics from all MPI processes into a single record and, in doing so, loses track of which process was the slowest. This situation has since been addressed in the Darshan 2.0.0 release. For shared files we therefore estimate the I/O time as the elapsed time between the beginning of the first open () call and the end of the last I/O operation on the file.

To verify the accuracy of this approach, we used the IOR benchmark. The results are shown in Table I. All IOR jobs used 4,096 processes and transferred a total of $1 \mathrm{TiB}$ of data to or from GPFS. Our examples used both shared files and unique files per process (notated as $\mathrm{N}-1$ and N-N, respectively). The aggregate performance derived from Darshan deviated by less than 3\% from the value reported by IOR in each case. Note that reads obtained higher performance than did writes, likely because current SAN configuration settings disable write-back caching but still cache read operations. The variance between $\mathrm{N}-1$ and $\mathrm{N}-\mathrm{N}$ is probably due to metadata operations because the IOR measurement includes both open and close time as well as the read and write time.

IOR, as configured in these examples, issues perfectly aligned $4 \mathrm{MiB}$ operations concurrently on all processes. Table I therefore also indicates the approximate maximum $\mathrm{MiB} / \mathrm{s} / \mathrm{CN}$ that can be observed on Intrepid. The general maximum performance is bound by the network throughput the ION can obtain. The BG/P tree network, which connects $\mathrm{CN}$ and ION, supports approximately $700 \mathrm{MiB} / \mathrm{s}$, which gives a theoretical maximum of $10.94 \mathrm{MiB} / \mathrm{s} / \mathrm{CN}$. The ION to storage network supports approximately $350 \mathrm{MiB} / \mathrm{s}$, which results in a theoretical maximum of $5.47 \mathrm{MiB} / \mathrm{s}$. With GPFS, a read workload can take advantage of read-ahead and caching to get above the storage network maximum. One final caveat is that the maximum possible $\mathrm{MiB} / \mathrm{s} / \mathrm{CN}$ rate will diminish as the total performance approaches the limit of the file system [1]. The theoretical maximum performance for a 40,960-node, 163,840process job is $1.59 \mathrm{MiB} / \mathrm{s} / \mathrm{CN}$. These metrics are not perfect representations of I/O performance; however, with the exception of three jobs that achieved unreasonably high measures by our estimate, the metric provides meaningful insight into relative performance for production jobs that cannot otherwise be explicitly instrumented. Further analysis indicates that the three outliers exhibited very sparse, uncoordinated I/O that did not fit the model.

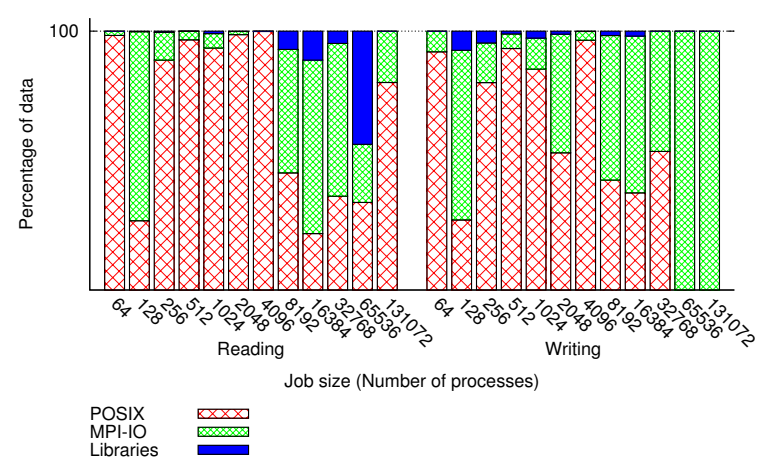

Fig. 2. Interfaces used by jobs as a function of job size.

\section{APPLICATION TRENDS AND I/O-INTENSIVE PROJECTS}

From January 23 to March 26, Intrepid executed 23,653 jobs that consumed a total of 175 million core-hours. These jobs were divided into 66 science and engineering projects (not counting maintenance and administration). Of these, 37 were INCITE projects, as described in Section II. The remainder were discretionary projects that are preparing INCITE applications or porting codes to the Blue Gene/P architecture. Of the total workload, Darshan instrumented 6,480 (27\%) of all jobs and 42 million (24\%) of all core-hours. At least one example from 39 of the 66 projects was captured.

\section{A. Overall application trends}

As part of our analysis we investigated the overall trends of the applications instrumented through Darshan on Intrepid. We were interested primarily in two characteristics. First, we wanted to discover which $\mathrm{I} / \mathrm{O}$ interfaces were used by applications at various job sizes. High-level interfaces such as PnetCDF and HDF5 ease the data management burden and provide data portability, but it has remained unclear how many applications utilize these interfaces and how much data is moved through them. MPI-IO provides useful optimizations for accessing the parallel file systems deployed at leadershipclass supercomputing centers, but applications may continue to use POSIX interfaces for a variety of reasons.

Second, we wanted a clearer understanding of the patterns of access in these applications. We focused on the type of file access at various job sizes, looking at the frequency and amount of I/O done to unique files (also known as $\mathrm{N}: \mathrm{N}$ ), shared files (N:1), and partially shared files (only a subset of processes perform $\mathrm{I} / \mathrm{O}$ to the file, $\mathrm{N}: \mathrm{M}$, where $\mathrm{M}<\mathrm{N}$ ). Intuitively, applications performing $\mathrm{I} / \mathrm{O}$ across a large number of processes will be more likely to use shared files in order to ease the file management burden that results when a large job writes 10,000-100,000 files, but it has remained unclear when applications choose shared files. Also, various studies have shown that at the largest job sizes, some file systems perform better under unique file workloads because lock contention is avoided [12], whereas others perform better under shared file workloads because they ease the metadata management 


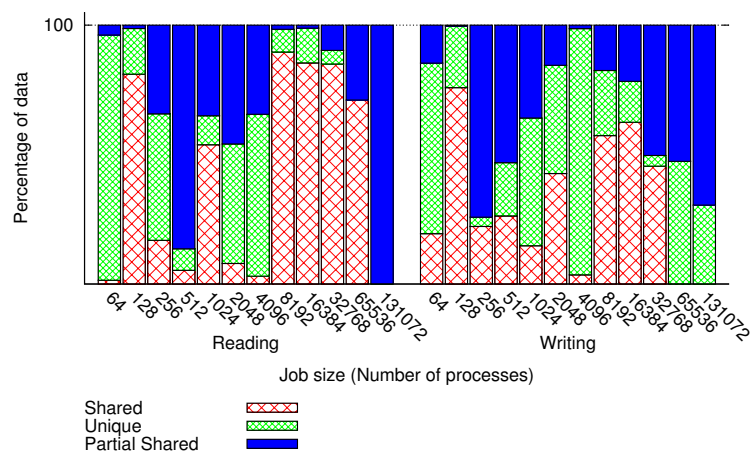

Fig. 3. I/O strategy used by jobs as a function of job size.

burden [1]. We wanted to see how application performance varied according to the strategy chosen.

Figures 2 and 3 give an overview of the I/O interfaces and access patterns, respectively, used by applications at various job sizes. We see in Figure 2 that the POSIX interfaces were used by the majority of jobs and performed the bulk of the $\mathrm{I} / \mathrm{O}$, especially for reading and at smaller process counts. Some applications used MPI-IO, particularly at the highest process counts and for applications that primarily wrote data. So few of the applications in our study used high-level libraries that they would not have been visible in the graph.

Figure 3 shows that the I/O strategy used by jobs varied considerably depending on the size of the job. Unique files were the most common access method for small jobs, whereas partially shared files were the most common access method for large jobs. In terms of quantity of data, most I/O was performed to files that were shared or partially shared. The widespread use of partially shared files indicates that applications are not relying on MPI-IO collective buffering optimization but, rather, are performing their own aggregation by writing and reading shared files from subsets of processes.

\section{B. I/O-intensive projects}

Not all the 39 projects captured by Darshan were significant producers or consumers of data. Figure 4 illustrates how much data was read and written by the ten projects that moved the most data via Darshan-enabled jobs. The projects are labeled according to their general application domain. The first observation from this figure is that that a few projects moved orders of magnitude more data than most others. The project with the highest I/O usage, EarthScience, accessed a total of $3.5 \mathrm{PiB}$ of data. Another notable trend in Figure 4 is that eight of the top ten projects read more data than was written. This is contrary to findings of previous scientific I/O workload studies [13]. By categorizing the data by project we see that the read/write mix varies considerably by application domain.

Table II lists coverage statistics and application programmer interfaces (APIs) used by each of the projects shown in Figure 4. Darshan instrumented over half of the corehours consumed by seven of the ten projects. NuclearPhysics, Chemistry, and Turbulence 3 were the exceptions and may

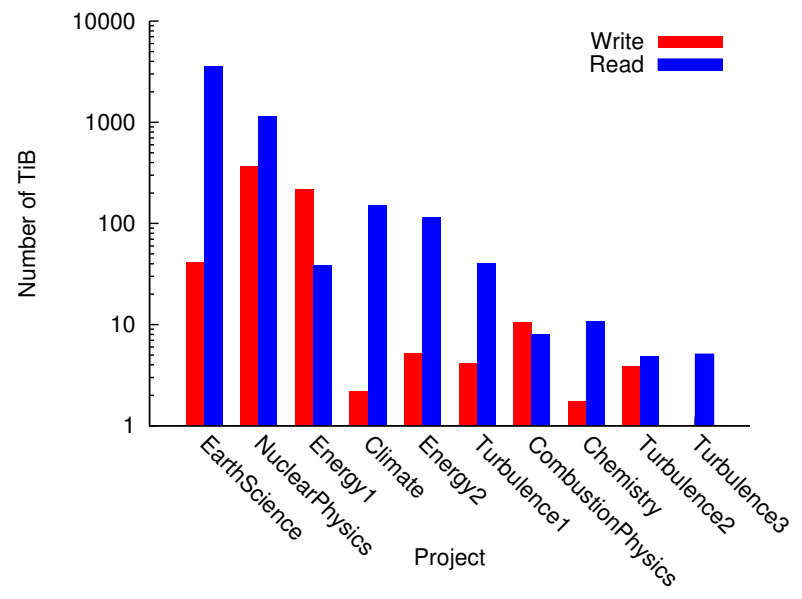

Fig. 4. Data moved per project in Darshan-enabled jobs.

have generated significantly more $\mathrm{I} / \mathrm{O}$ activity than is indicated by Figure 4 . The fourth column of Table II shows which APIs were used directly by applications within each project. $\mathrm{P}$ represents the POSIX open() interface, $\mathrm{S}$ represents the POSIX stream fopen ( ) interface, M represents MPI-IO, and $\mathrm{H}$ represents HDF5. Every project used at least one of the two POSIX interfaces, while four projects also used MPI-IO. Energy1 notably utilized all four of HDF5, MPI-IO, POSIX, and POSIX stream interfaces in its job workload.

This subset of projects also varies in how many files are used. Figure 5 plots the number of files accessed by application run according to its processor count for our ten most I/O-intensive projects. If several application instances were launched within a single job (as is common on Intrepid), each instance is shown independently. Reinforcing Figure 3, we see four rough categories: applications that show an N:N trend, ones that show an $\mathrm{N}: 1$ trend, a group in the middle exemplified by Turbulence 3 that are subsetting (N:M), and a fourth category of applications operating on no files. The large number of application runs that operated on zero files is surprising. Darshan does not track standard output or standard error. One possible explanation is that projects appear to run a few debug jobs to run diagnostic or preliminary tests that write results only to standard out or standard error and then

TABLE II

DARSHAN COVERAGE OF HIGHLIGHTED PROJECTS

\begin{tabular}{lrrr}
\hline Project & $\begin{array}{r}\text { Job } \\
\text { Coverage }\end{array}$ & $\begin{array}{r}\text { Core-Hour } \\
\text { Coverage }\end{array}$ & APIs $^{a}$ \\
\hline EarthScience & $779 / 1488$ & $10.9 / 11.8 \mathrm{M}$ & $\mathrm{S}, \mathrm{P}$ \\
NuclearPhysics & $1653 / 6159$ & $11.3 / 62.7 \mathrm{M}$ & $\mathrm{P}$ \\
Energy1 & $994 / 1340$ & $3.7 / 5.7 \mathrm{M}$ & $\mathrm{H}, \mathrm{M}, \mathrm{S}, \mathrm{P}$ \\
Climate & $32 / 130$ & $2.0 / 3.3 \mathrm{M}$ & $\mathrm{S}$ \\
Energy2 & $384 / 1433$ & $3.9 / 4.4 \mathrm{M}$ & $\mathrm{S}, \mathrm{P}$ \\
Turbulence1 & $242 / 467$ & $2.6 / 4.6 \mathrm{M}$ & $\mathrm{M}, \mathrm{S}, \mathrm{P}$ \\
CombustionPhysics & $15 / 42$ & $1.8 / 2.4 \mathrm{M}$ & $\mathrm{S}, \mathrm{P}$ \\
Chemistry & $28 / 144$ & $0.1 / 0.6 \mathrm{M}$ & $\mathrm{S}$ \\
Turbulence2 & $70 / 157$ & $0.3 / 0.3 \mathrm{M}$ & $\mathrm{M}, \mathrm{P}$ \\
Turbulence3 & $172 / 418$ & $0.1 / 13.3 \mathrm{M}$ & $\mathrm{M}, \mathrm{S}, \mathrm{P}$ \\
\hline
\end{tabular}

${ }^{a} \mathrm{P}=$ POSIX, $\mathrm{S}=$ POSIX stream, $\mathrm{M}=$ MPI-IO, $\mathrm{H}=$ HDF5 


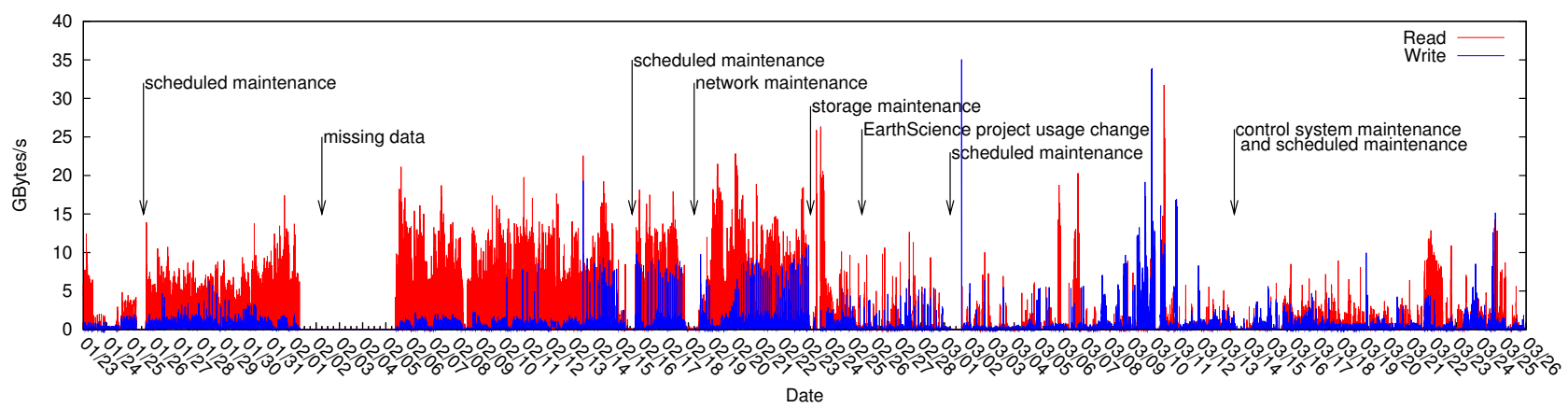

Fig. 6. Aggregate throughput on one-minute intervals.

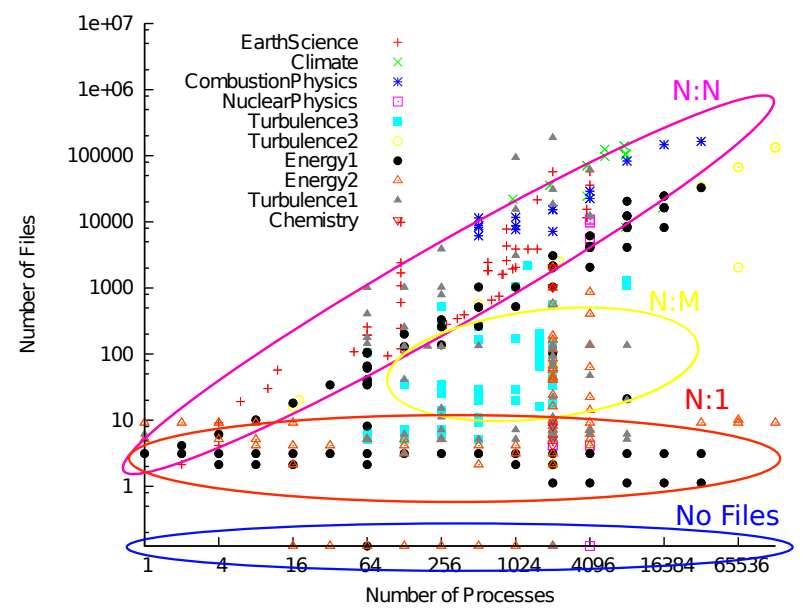

Fig. 5. Number of files written by size of application run for our ten most I/O-intensive projects.

proceed to run "real" jobs.

Of the N:N applications, some access as many as 100 files per process. Programs accessing multiple files per process might need special attention when scaling to full-machine runs because of challenges in metadata overhead and file management.

Figure 5 also demonstrates that some projects have both $\mathrm{N}: 1$ and $\mathrm{N}: \mathrm{N}$ jobs. Perhaps the clearest example is NuclearPhysics, the purple rectangle, about which more will be said in Section V-B.

\section{Storage Utilization}

The previous section provided an overview of how applications and jobs of varying sizes interacted with the storage system. In this section we investigate how this interaction translates into utilization at the storage device and file system level.

Figure 6 shows the combined aggregate throughput at the block device level of Intrepid's main storage devices from January 23 to March 26. This includes both GPFS and PVFS activity. It also includes interactive access from login nodes as well as analysis access from the Eureka visualization cluster. Notable lapses in storage activity have been correlated with various maintenance windows and labeled accordingly. There were four notable scheduled maintenance days, as well as three unplanned maintenance windows due to network, storage, or control system issues. Note that the data from 9:00 am February 1 to 10:00 am February 5 was lost because of administrative error, but the system was operating normally during that time.

The peak read throughput achieved over any one minute interval was $31.7 \mathrm{GiB} / \mathrm{s}$, while the peak write throughput was $35.0 \mathrm{GiB} / \mathrm{s}$. In previous work, we found that end-to-end throughput on this system varied depending on the access pattern [1]. In that study we measured maximum read performance from 33 to $47 \mathrm{GiB} / \mathrm{s}$ and maximum write performance from 30 to $40 \mathrm{GiB} / \mathrm{s}$, both using PVFS. The system did not quite reach these numbers in practice during the interval shown in Figure 6. This may be caused by SAN hardware configuration changes since the previous study. Our previous study also took advantage of full system reservations during Intrepid's acceptance period, with no resource contention.

From the iostat logs we can also calculate the amount of data moved over various time intervals. An average of $117.1 \mathrm{TiB}$ were read per day, and $31.5 \mathrm{TiB}$ were written per day. A total of $6.8 \mathrm{PiB}$ and $1.8 \mathrm{PiB}$ were read and written over the study interval, not counting the four missing days of data. Although reads made up $78.8 \%$ of all activity over the course of the study, this was largely due to the behavior of a single project. The EarthScience project was noted in Section III for having the most read-intensive workload of all projects captured by Darshan. It read over $3.4 \mathrm{PiB}$ of data during the study, or approximately half the total read activity on the system. We investigated that project's usage activity in scheduler logs and found that it significantly tapered off around February 25. This corresponds to a visible change in the read/write mixture at the same time in Figure 6. For the following two weeks, reads accounted for only $50.4 \%$ of all I/O activity.

One explanation for the unexpectedly high level of read activity is that users of Intrepid do not checkpoint as frequently as one might expect. The maximum job time allowed by scheduling policy is 12 hours, which is significantly less than the mean time to interrupt of the machine (especially if boot time errors are excluded). Many applications therefore checkpoint only once per run unless they require more frequent checkpoints for analysis purposes. We also note that some fraction of read activity was triggered by unaligned write 


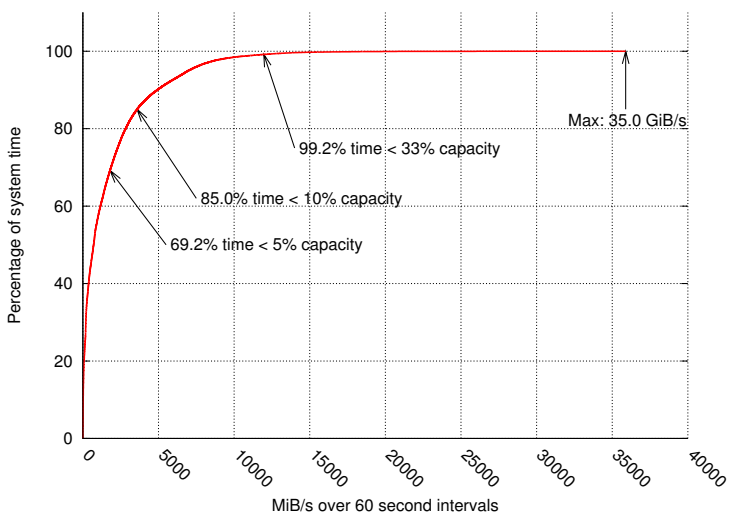

Fig. 7. Cumulative distribution of aggregate storage throughput.

accesses at the application level. Both GPFS and PVFS must ultimately perform read/modify/write operations at the block level in order to modify byte ranges that do not fall precisely on block boundaries. As we will see in Section V, unaligned access is common for many applications.

Figure 6 suggests that the I/O activity is also bursty. To quantify this burstiness, we generated a cumulative distribution function of the combined read and write throughput on the system for all 63,211 one-minute intervals recorded from iostat. The result is shown in Figure 7. The average total throughput was $1,984 \mathrm{MiB} / \mathrm{s}$. The peak total throughput was $35,890 \mathrm{MiB} / \mathrm{s}$. For $98 \%$ of the time, the I/O system was utilized at less than $33 \%$ of peak I/O bandwidth. This performance matches with the common understanding of burstiness of $\mathrm{I} / \mathrm{O}$ at these scales. Because leadership-class I/O systems are provisioned to provide a very high peak bandwidth for checkpointing, during computation phases the I/O system will be mostly idle.

The duration of the idle periods varied considerably. Table III summarizes the duration of idle periods if we define an idle period as any time in which the aggregate throughput was less than $5 \%$ of peak for consecutive 60 -second intervals. Idle periods lasting less than 10 minutes by this definition are quite common, but the majority of the idle time is coalesced into much longer periods. Over $37 \%$ of the total system time was spent in idle periods that lasted at least one hour. The longest example lasted 19 hours on March 1, which was a scheduled

TABLE III

DISTRIBUTION OF TIME PERIODS IN WHICH THE AGGREGATE THROUGHPUT WAS LESS THAN 5\% OF PEAK FOR CONSECUTIVE 60 SECOND INTERVALS

\begin{tabular}{rrrr}
\hline $\begin{array}{r}\text { Duration } \\
\text { (minutes) }\end{array}$ & Count & $\begin{array}{r}\text { Cumulative } \\
\text { Minutes }\end{array}$ & $\begin{array}{r}\text { Percentage } \\
\text { of Total Time }\end{array}$ \\
\hline 1 & 1420 & 1420 & $1.7 \%$ \\
$2-5$ & 2259 & 7053 & $8.4 \%$ \\
$6-10$ & 775 & 5882 & $7.0 \%$ \\
$11-20$ & 383 & 5530 & $6.6 \%$ \\
$21-30$ & 104 & 2581 & $3.1 \%$ \\
$31-40$ & 50 & 1756 & $2.1 \%$ \\
$41-50$ & 30 & 1369 & $1.6 \%$ \\
$51-60$ & 19 & 1052 & $1.3 \%$ \\
$>60$ & 169 & 30935 & $37.1 \%$ \\
\hline
\end{tabular}

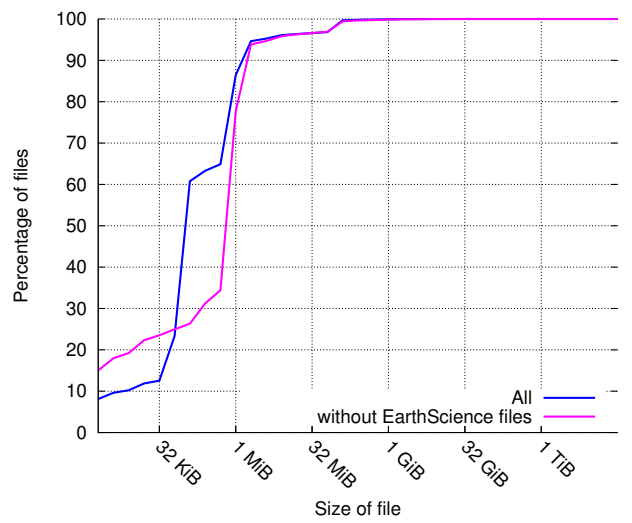

Fig. 8. Cumulative distribution of file sizes, March 2010.

maintenance day for Intrepid. If we limit the definition of idle periods to only those in which no disk activity was observed at all, then minute-long idle periods are much less common. We observed 935 such intervals, which accounted for just over $1 \%$ of the total system time. This stricter definition of idle time would be better investigated by using tools with smaller granularity [14] in order to capture shorter intervals.

We believe that both definitions of idle time are relevant for different aspects of storage system design. Time in which the storage system is mostly idle presents an opportunity for file systems to leverage unused capacity for autonomous storage activity, while time in which parts of the storage system are completely idle may be more useful for component-level optimizations.

\section{A. File system contents}

Despite the quantity of data that was transferred through the storage system on Intrepid, a surprising amount of it was stored in relatively small files at the file system level. Figure 8 illustrates the cumulative distribution function of file sizes in March 2010. The most popular size range was $64 \mathrm{KiB}$ to 128 $\mathrm{KiB}$, with over 71 million files. Of all files, $86 \%$ were under $1 \mathrm{MiB}, 95 \%$ were under $2 \mathrm{MB}$, and $99.8 \%$ were under 128 MB. The largest file was 16 TiB.

Closer investigation, however, revealed that a single project, EarthScience, was significantly altering the file size characteristics. Figure 8 shows that without EarthScience's files, the most popular file size would have been $512 \mathrm{KiB}$ to $1 \mathrm{MiB}$ and only $77 \%$ of the files would have been under $1 \mathrm{MiB}$ in size.

We can also observe how the file systems changed over the study interval by comparing fsstats results from the beginning and end of the two-month study. Table IV shows growth of the primary file systems on Intrepid, in terms of both total capacity

TABLE IV

FILE SYSTEM STATISTICS

\begin{tabular}{lrrrr}
\hline & \multicolumn{2}{c}{ Total } & \multicolumn{2}{c}{ EarthScience } \\
\hline Date & Usage & Files & Usage & Files \\
\hline January & $1.14 \mathrm{~PB}$ & $96.2 \mathrm{M}$ & $274.4 \mathrm{~TB}$ & $12.3 \mathrm{M}$ \\
March & $1.28 \mathrm{~PB}$ & $191.4 \mathrm{M}$ & $295.1 \mathrm{~TB}$ & $96.2 \mathrm{M}$ \\
Change & $+12.3 \%$ & $+99.0 \%$ & $+7.5 \%$ & $+682.1 \%$ \\
\hline
\end{tabular}




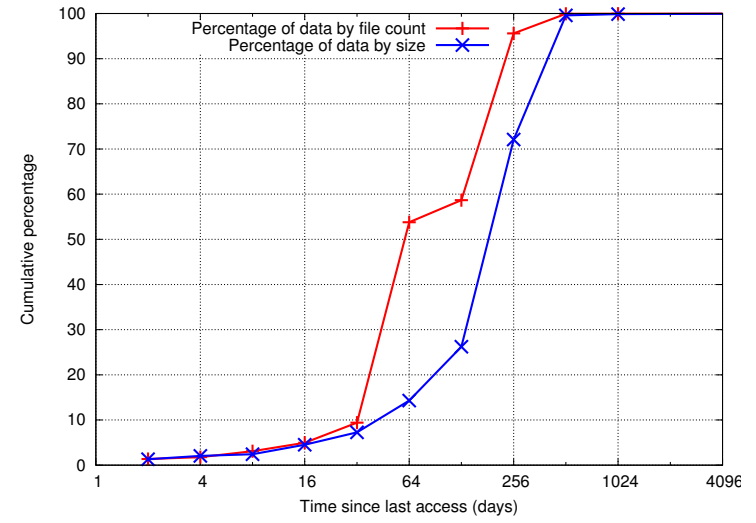

(a) Access time

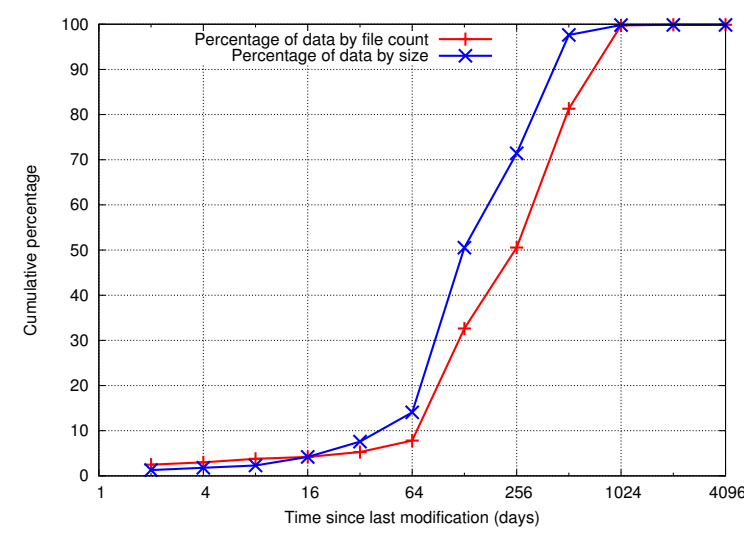

(b) Modification time

Fig. 9. Cumulative distribution of file access and modification time

and number of files. Over the study period, the number of files doubled. Just as in the static analysis, however, this growth was largely the result of the EarthScience project data. EarthScience was responsible for $88 \%$ of the additional files created during the study period but generated only $15 \%$ of the new data by capacity. If the number of files continues to increase at the same rate, the file system will reach 1 billion files in September 2011.

\section{B. File age and overwriting data}

Although a large number of files are stored on Intrepid, not all of them are accessed frequently. Figure 9(a) shows a cumulative distribution plot of the last access time for each file on Intrepid in March 2010. Shown are the cumulative percentage based on the number of files and on the amount of data in each file. By either metric, over $90 \%$ of the data on Intrepid has not been accessed in at least a month. Moreover, although $55 \%$ of all files have been accessed in the past 64 days, those files accounted for only $15 \%$ of the data stored on the file system by volume. Figure 9(b) shows similar data for the modification time for the same files: $8 \%$ of all files were written in the past 64 days, but those files accounted for $15 \%$ of the data on the system by volume. The most pronounced difference between Figure 9(a) and Figure 9(b) is that the lines for file count and file size percentages are reversed. This

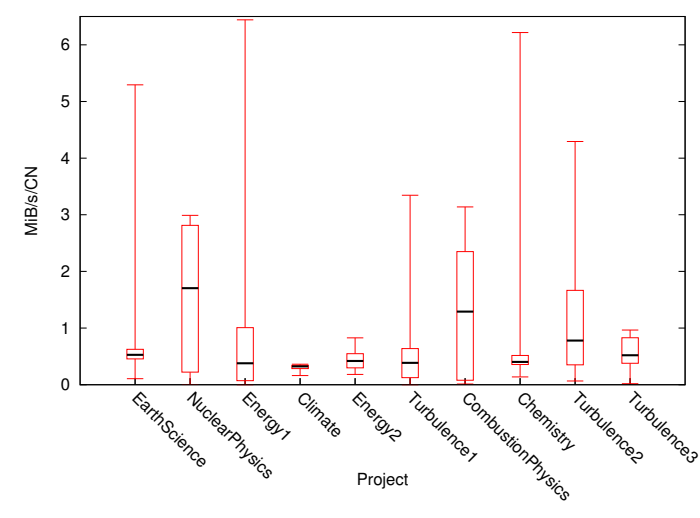

Fig. 11. Performance per project in large Darshan-enabled jobs (EarthScience outliers are not shown). Peak performance falls between $1.6 \mathrm{MiB} / \mathrm{s} / \mathrm{CN}$ and $10.9 \mathrm{MiB} / \mathrm{s} / \mathrm{CN}$, depending on job size and other active jobs.

suggests that small files tend to be read more frequently than large files on Intrepid.

The modification data and access time data suggest that files are rarely overwritten once they are stored on the file system. Darshan characterization supports this observation. We found that of the 209.5 million files written by jobs instrumented with Darshan, $99.3 \%$ either were created by the job or were empty before the job started.

We note that the jobs characterized by Darshan wrote more files in the two-month study than were actually present in the file system at the end of the study. This fact, in conjunction with the earlier observations, indicates that files either are deleted within a relatively short time frame or are stored unchanged for extended periods of time. These characteristics would be beneficial to algorithms such as replication, compression, and hierarchical data management that can take advantage of infrequently modified files to improve efficiency.

\section{I/O CHARACTERISTICS BY PROJECT}

We have established that the I/O workload on Intrepid consists of a variety of access patterns and file usage strategies and that the underlying storage system experiences bursts of I/O demand. In this section we explore in greater detail how storage access characteristics vary by application domain and how those characteristics correlate with I/O performance.

Figure 11 is a box plot of performance measured by using the $\mathrm{MiB} / \mathrm{s} / \mathrm{CN}$ metric outlined in Section II-D. We have filtered the jobs captured by Darshan to include only those that used at least 1,024 processes and moved at least $500 \mathrm{MiB}$ of data. This approach eliminates noise from jobs that moved trivial amounts of data. All statistics shown in the remainder of this study are filtered by the same criteria. For each project in Figure 11, we have shown the minimum, median, and maximum, as well as the Q1 and Q3 quartiles. Some projects exhibited very consistent performance, whereas others varied over a relatively wide range. Very few jobs from any project approached the maximum values established in Section II-D.

Table V summarizes a set of key storage access characteristics as averaged across jobs within that project. The MiB/s/CN 
TABLE V

AVERAGE I/O CHARACTERISTICS OF LARGE JOBS BY PROJECT

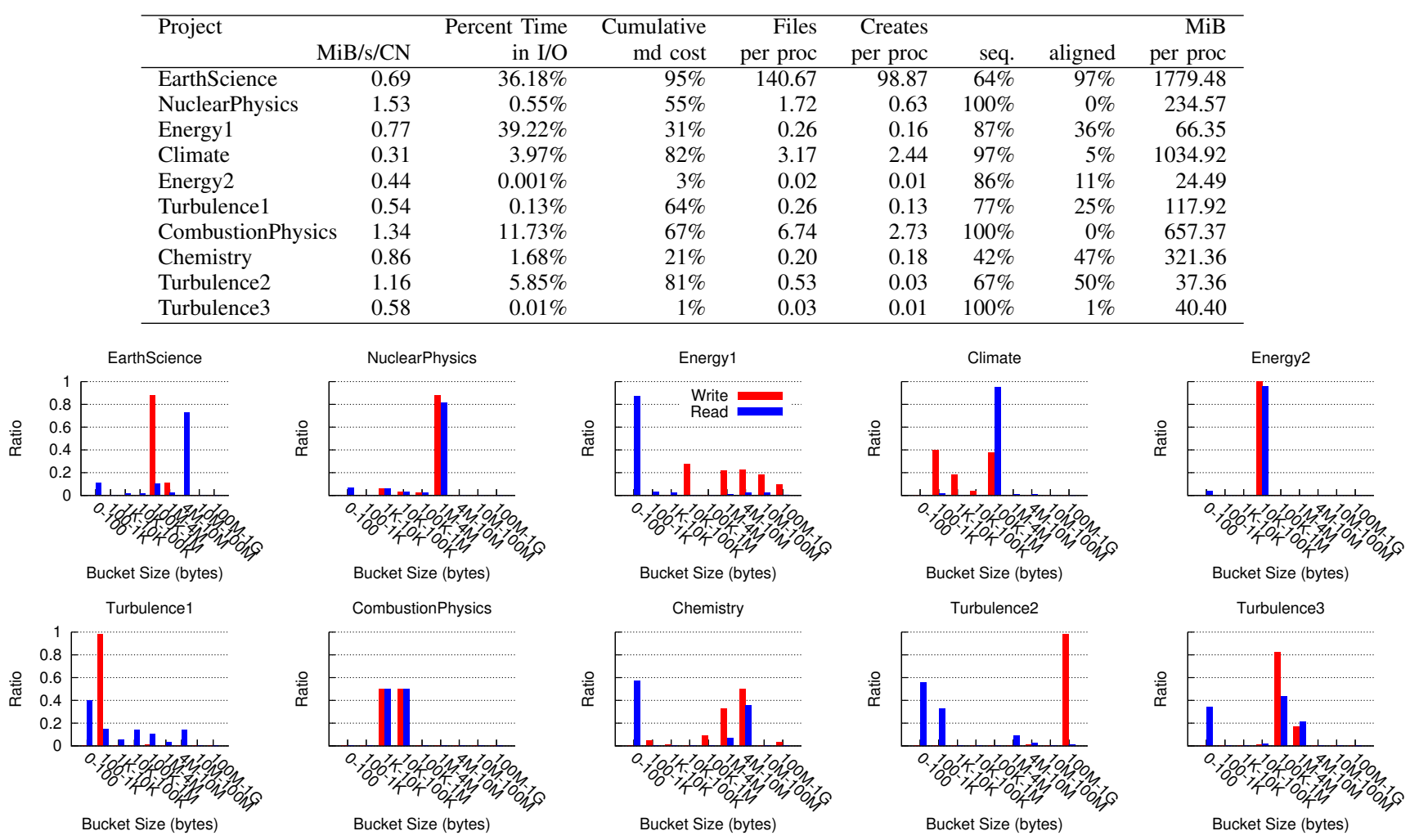

Fig. 10. Access sizes of large jobs by project.

and metadata overhead are computed as described in Section II-D. The second column shows the percentage of overall job run time that was spent performing I/O operations. The third column shows the percentage of that $\mathrm{I} / \mathrm{O}$ time that was spent performing metadata operations rather than read () or write () operations. This value is much higher than expected in some cases because of the GPFS file system flushing writes to small files at close () time, since Darshan counts all close () operations as metadata. The other columns show the number of files accessed and created per MPI process, the percentage of sequential and aligned accesses, and the amount of data moved per process. Often accesses by a given process are highly sequential, as has been seen in previous studies [13]. Figure 10 illustrates in greater detail the access sizes used by each project; histograms represent the percentages of accesses that fell within each range.

In the remainder of this section we will refer to Table $\mathrm{V}$ and Figure 10 as we explore the characteristics of each project in greater depth.

\section{A. EarthScience}

The EarthScience project has already featured prominently in previous sections because it dominated both the read activity and number of files stored on Intrepid during the study interval. Despite the high level of I/O usage, however, EarthScience ranked near the low range of median I/O performance. Other than the three outliers discussed earlier, the performance is consistent, with an interquartile range (IQR) of only 0.17
$\mathrm{MiB} / \mathrm{s} / \mathrm{CN}$. Further inspection indicated that the EarthScience workload is dominated by 450 nearly identical jobs, each of which utilized 4,096 processes. These jobs were often further subdivided into a sequence of up to 22 repeated instances of the same application within a job allocation. Each instance accessed approximately 57,000 files, leading some jobs to access a total of more than 1 million distinct files over the lifetime of the job.

The EarthScience project read over 86 times more data than it wrote. The data that it did write, however, was broken into a large number of newly created files. Of the 141 files accessed per process on average, 99 were created and written by the job itself. As noted in Section IV, this project alone contributed over 96 million files to the 191.4 million stored on Intrepid at the end of the study. The direct result of splitting data into so many files is that each job spent more of its I/O time performing metadata operations than actually reading or writing application data. Over $20 \mathrm{TiB}$ of data were written into files averaging $109 \mathrm{KiB}$ each in size, leaving the file system little opportunity to amortize metadata overhead. The apparent metadata cost is exaggerated somewhat by I/O time that is attributed to close () rather than write (), but that doesn't change the fact this metadata overhead is a limiting factor in overall I/O efficiency for the project.

\section{B. NuclearPhysics}

NuclearPhysics exhibited the widest IQR of job performance of any of the ten most I/O-intensive projects. This 
variability was not caused by fluctuations in performance of a single application. Two applications with different I/O characteristics were run by users as part of this project. In one set, 809 nearly identical jobs accounted for the upper quartile and were among the most efficient of any frequently executed application during the study. In the other set, 811 jobs accounted for the lower quartile. This example illustrates that access characteristics may vary significantly even across applications from the same domain on the same system.

The faster of the two applications utilized a partially shared file access pattern (N:M) and was atypical among jobs observed in this study because many of its files were both read and written to during the same job. The metadata overhead of creating and writing multiple files was amortized by the amount of $\mathrm{I} / \mathrm{O}$ performed to each file. An example job read $1.38 \mathrm{TiB}$ of data and wrote $449.38 \mathrm{GiB}$ of data. This job is also a clear example of a behavior that was first speculated in Section III-A, namely, that some applications are implementing their own form of $\mathrm{I} / \mathrm{O}$ aggregation rather than using the collective functionality provided by MPI-IO. This particular application used POSIX exclusively and was run with 4096 processes, but the first 512 MPI ranks performed all of the I/O for each job.

The slower of the two applications that dominated this project presents an example of an application that performs "rank 0" I/O, in which a single process is responsible for all of the I/O for the job. In this case the jobs were either 2,048 or 4,096 processes in size. The fact that all $\mathrm{I} / \mathrm{O}$ was performed by a single rank resulted in a $\mathrm{MiB} / \mathrm{s} / \mathrm{CN}$ score as low as 0.2 in most cases. At first glance this appears to be very poor $\mathrm{I} / \mathrm{O}$ behavior, but in practice these jobs read only $4 \mathrm{GiB}$ of data, and the time to read that data with one process often constituted only $1 \%$ of the run time for this application. So while the storage access characteristics were poor, it will likely not be a significant problem unless the application is scaled to a larger problem size. This application accounted for the earlier observation in Figure 5 that NuclearPhysics exhibited both $\mathrm{N}: \mathrm{M}$ and $\mathrm{N}: 1$ styles of access patterns.

\section{Energyl}

The performance fluctuation in the Energy 1 project results from variations within a single application that used different file systems, job sizes, APIs, data sizes, and file sharing strategies. Discussions with the scientists involved in the project revealed that this behavior was the result of experimental I/O benchmarking and does not represent production application behavior. However, it was interesting to capture an applicationoriented I/O tuning experiment in progress.

\section{Climate}

The Climate project executed 30 jobs. The jobs tended to use co-processor mode, which means 2 MPI processes per node with 2 threads per MPI process. The application performance was likely dominated by three factors. First, each process created two files, translating to a higher metadata overhead. Second, the application performed a seek for every
TABLE VI

Turbulence1 Performance by Job

\begin{tabular}{lrrrr}
\hline Job & Procs & $\begin{array}{r}\text { Performance } \\
(\mathrm{MiB} / \mathrm{s} / \mathrm{CN})\end{array}$ & $\begin{array}{r}\text { Metadata } \\
(\%)\end{array}$ & $\begin{array}{r}\text { Key Access } \\
\text { Size }\end{array}$ \\
\hline A & 8192 & 2.92 & $28 \%$ & $74 \mathrm{KiB}$ \\
$\mathrm{B}$ & 2048 & 1.12 & $2 \%$ & $20 \mathrm{~B}(\mathrm{w})$ \\
$\mathrm{C}$ & 1024 & 0.64 & $6 \%$ & $<20 \mathrm{~B}(\mathrm{w})$ \\
$\mathrm{D}$ & 4096 & 0.38 & $41 \%$ & $<20 \mathrm{~B}(\mathrm{w})$ \\
$\mathrm{E}$ & 2048 & 0.11 & $36 \%$ & $<700 \mathrm{~B}(\mathrm{r}+\mathrm{w})$ \\
$\mathrm{F}$ & 32768 & 0.0009 & $1 \%$ & $4 \mathrm{~B}(\mathrm{r})$ \\
\hline
\end{tabular}

$\mathrm{read} / \mathrm{write}$ operation. All seeks need to be forwarded to the ION to be processed, making the calls unusually expensive relative to a cluster system; $82 \%$ of I/O time was spent in metadata. Third, write operations were only $64 \mathrm{KiB}$ when the file system block size was $4 \mathrm{MiB}$. Writes this small are not efficient on this system.

\section{E. Energy 2}

The Energy 2 project executed 58 jobs at one of two sizes, 2,048 or 4,096 processes. The overall time spent in I/O as a whole was very small (less than $1 \%$ ). The I/O performance of this project was low compared with the others' performance, even though it had low overhead for metadata. The performance loss was due to small independent writes (less than $10 \mathrm{KiB}$ ) that occurred only on rank 0 . This project does utilize a single, shared file for reading, in which all processes read significantly more bytes than are written, and at a larger access size, producing very good performance. Given the small amount of time spent in I/O as compared to the overall application run-time and the minimal number of bytes, maximizing the write performance doesn't seem to be a priority.

\section{F. Turbulence1}

The Turbulence project sample contained 118 diverse jobs. The jobs were run by three users, with process counts between 1,024 and 32,768. Each user ran a few different applications, which led to a wide performance range for all applications. Table VI details example jobs at the different performance scales.

All of these jobs had a common I/O profile. Each application used shared files as well as unique files on a fileper-process basis. For applications that needed to read/write more substantial amounts of a data, a single shared file was used with a $4 \mathrm{MiB}$ access size. The bulk of the accesses, however, involved very small read or write operations. As a result, performance was determined by the effectiveness of either collective I/O or POSIX stream operations to combine these small I/O operations into larger requests. The fastest job performed the bulk of its I/O to shared files using POSIX read operations using the fast scratch file system. The access size was not particularly large but probably benefited from the GPFS read-ahead caching. The slowest application used a large number of independent POSIX reads of a very small access size, on the order of four bytes to the slower home file system. 


\section{G. CombustionPhysics}

The CombustionPhysics project comprised only 11 jobs in the sample based on the selection criteria. Within those 11 were a wide variety of different-sized jobs. The size of the job had a significant impact on the I/O rate.

This project appeared to be studying strong scaling since the total number of bytes transferred for each job was similar regardless of size. Hence, the bytes per process transferred were smaller at each larger job size. At the two smaller node counts $(1,024$ and 2,048) the total I/O time for the job was small $(<1 \%)$ compared with the total compute time. At the 4,096 node count, however, the total I/O time became $40 \%$ of the run time, and the percentage of time spent in metadata exploded to $35 \%$. At each larger node count the percentage of I/O time spent in metadata increased, eventually topping out at $99 \%$.

Table $\mathrm{V}$ indicates that this project created about three files per process on average. This I/O strategy did not scale well on Intrepid for higher processor counts and smaller amounts of data per process (see Table VII). In Section VI we will revisit this project and evaluate the impact of various I/O tuning strategies that were guided by the findings of our integrated I/O characterization methods.

\section{H. Chemistry}

All the data captured for the Chemistry project corresponds to a single application. The bulk data accesses from the application were all perfectly aligned to the file system at $4 \mathrm{MiB}$. The metadata overhead was also low, because the majority of jobs accessed fewer than 10 total files regardless of job size. The I/O efficiency is poor despite these characteristics, however. The reason is that (with one exception) all of the Chemistry jobs captured by Darshan performed I/O exclusively from a single process, regardless of the size of the job. These jobs achieved performance similar to the lower quartile jobs of the NuclearPhysics project that utilized the same strategy.

One instance of the same application was executed with notably different characteristics. The job size in that case was 2,048 , and half of the processes were involved in performing I/O. As in the upper quartile NuclearPhysics cases, the application appears to be manually performing aggregation on behalf of the other processes, as no MPI-IO is involved. The 1,024 I/O tasks combined to read $10 \mathrm{TiB}$ of data and write $1.35 \mathrm{TiB}$ of data. A unique file was used by each process, and all data was perfectly aligned. The application was therefore able to sustain I/O for an extended period with no significant metadata or misalignment overhead. This job achieved the highest observed efficiency of jobs analyzed in the case studies.

\section{Turbulence 2}

The Turbulence 2 project illustrates another example where the job variability arose from differences in the performance of a single application at different scales. The jobs took very little run time, with several examples executing for less than one
TABLE VII

CombustionPhysics Performance by Job Size

\begin{tabular}{lrr}
\hline Nodes & Jobs & $\begin{array}{r}\text { Performance } \\
\text { MiB/s/CN }\end{array}$ \\
\hline 1024 & 3 & 3.0 \\
2048 & 3 & 1.3 \\
4096 & 2 & 0.36 \\
8192 & 1 & 0.08 \\
16384 & 1 & 0.03 \\
32768 & 1 & 0.01 \\
\hline
\end{tabular}

minute. There is an unusual mix of access sizes, as illustrated in Figure 10. Writes were dominated by very large access sizes, but many reads were less than 100 bytes each. This strategy performed best at relatively small job sizes of 2,048 or 2,304 processes. The same application did not fare was well when scaled up to 65,536 or 131,072 processes, though the run time was still only a few minutes. This application used MPI-IO but did not leverage collective operations or derived datatypes. The I/O-intensive jobs in this project may have been the result of a benchmarking effort, as only 40 jobs met the filtering criteria used in this section. All 40 were the same application executed with different parameters and at different scales.

\section{J. Turbulence3}

The Turbulence 3 project consisted of 49 jobs. There were a few common sizes of 1,024, 1,600, and 2,048 processes as well as two jobs of 8,192 processes. The jobs have a similar pattern of I/O. There is a mix of MPI independent reads and writes at a specific request size. The I/O also occurs only from a subset of the MPI ranks, either 4 or 8 ranks. The lowestperforming job used $16 \mathrm{KiB}$ MPI-IO independent reads and writes. The performance increased as jobs used larger request sizes, going up to $320 \mathrm{KiB}$ and $512 \mathrm{KiB}$ request sizes. The highest performing job used a $320 \mathrm{KiB}$ request size but had more than double the number of reads as writes. The reads would be able to take advantage of GPFS read-ahead and caching.

\section{APPLICATION TUNING CASE STUDY}

At the conclusion of the I/O study, we selected the CombustionPhysics project from Section V-G as a case study of how continuous characterization can be applied to tuning a specific application. As noted earlier, production jobs from this project ranged from 1,024 to 32,768 nodes but achieved progressively worse I/O performance as the scale increased. This application used OpenMP with four threads per node to process an AMR data set ranging from $2^{10}$ to $2^{13}$ data points. To simplify the tuning process, we decided to investigate the I/O of a similar but smaller application example from the CombustionPhysics project. This target application utilized the same OpenMP configuration and the same I/O strategy and likewise achieved poor I/O performance at scale. However, its data set is a uniform $2^{9}$ mesh that produces fixed-size checkpoints of approximately $20 \mathrm{GiB}$ each. We focused on the 8,192 node $(32,768$ cores) example of this application as a test case and configured it to generate two checkpoints. 


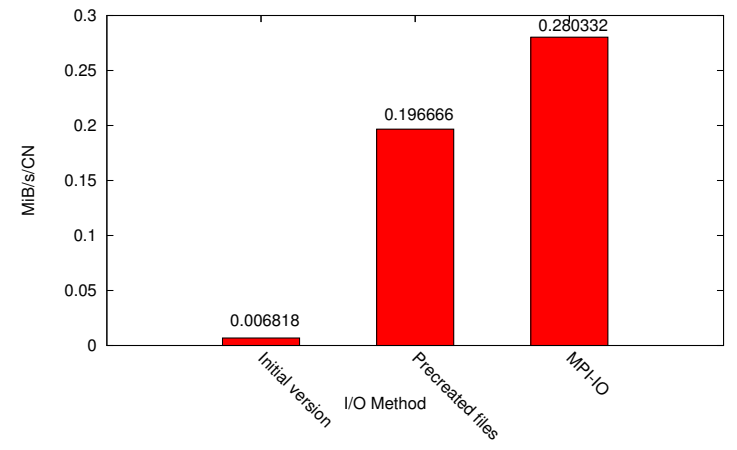

Fig. 12. CombustionPhysics per node performance with 8,192 nodes $(32,768$ cores).

This application was originally tuned for a highperformance computing system that achieved optimal performance by using a unique file for the checkpoint data on each node. As in examples shown earlier in this work, however, we found that on Intrepid this approach led to I/O behavior that was dominated by metadata overhead. According to Darshan, the total I/O time to dump $40 \mathrm{GiB}$ of data was approximately 728 seconds, nearly all of which was attributed to metadata activity. To confirm this observation, we precreated the output files for the application and measured the change in $\mathrm{I} / \mathrm{O}$ performance when file creation cost was eliminated from the run time. This reduced the total I/O time to 25 seconds, a factor of 28 improvement over the original execution. Such an approach is not feasible for production use, however: file precreation is a time-consuming process, and the number of checkpoints that will be generated is not known in advance.

To reduce the metadata overhead in a more practical manner, we decided to modify the application to dump its checkpoint data to a single, shared file. Rather than dumping data using independent POSIX operations, however, we updated the application to use MPI-IO collective operations. As a result, the application not only reduced metadata overhead but also enabled a range of transparent MPI-IO optimizations. Of particular importance for strong scaling algorithms, MPI-IO can aggregate access to shared files in order to mitigate the effect of smaller writes at scale. Collective MPI-IO routines also introduce precise block alignment, which has been shown to be an important factor in GPFS performance [15], [12].

Figure 12 shows the per node I/O performance achieved by all three versions of the test application with two checkpoints. The MPI-IO version achieved a factor of 41 improvement, dumping two time steps in approximately 17 seconds. Darshan analysis confirmed that all application-level writes were between 1 and $4 \mathrm{MiB}$, as in the original example. However, MPIIO aggregated these into $16 \mathrm{MiB}$ writes at the file system level. The total number of write operations processed by the file system was reduced from 16,388 to 4,096. This, in conjunction with improved block alignment and a dramatic reduction in the number of files created, resulted in more efficient use of available resources and faster turnaround for science runs.

\section{RELATED WORK}

A number of past studies have investigated the I/O access patterns of scientific applications. Nieuwejaar et al. initiated the influential Charisma project in 1993 to study multiprocessor I/O workloads [13]. This culminated in an analysis of three weeks of data from two high-performance computing systems with up to 512 processes. Their study identified access pattern characteristics and established terminology to describe them. Smirni and Reed analyzed five representative scientific applications with up to 64 processes [16]. The Pablo environment [17] was used for trace capture in that work. While both Charisma and Pablo measured application characteristics in a manner similar to our work, neither was performed at a comparable scale or correlated to system-level I/O activity. Wang et al. investigated synthetic benchmarks and two physics applications with up to 1,620 processes and found similar results [18]. Uselton et al. developed a statistical approach to I/O characterization in a more recent study [19]. They leveraged IPM [20] for the raw trace capture of two scientific applications with up to 10,240 processes on two different platforms. Statistical techniques were then used to identify and resolve I/O bottlenecks in each application. These studies utilized complete traces that focused on specific applications rather than on a general production workload.

Kim et al. performed a workload characterization of a 10 PiB storage system at Oak Ridge National Laboratory that provides storage for over 250 thousand compute cores [14]. They observed and modeled production disk controller characteristics such as bandwidth distribution, the correlation of request size to performance, and idle time. These characteristics were not correlated back to application level behavior, however.

Other recent system-level studies have focused on large network file systems. In an investigation of two CIFS file systems that hosted data for 1,500 industry employees, Leung et al. discovered a number of recent trends in I/O behavior [6]. Anderson presented a study of NFS workloads with up to 1,634 clients [5]. These studies were similar in scope to our work but were not performed in a high-performance computing environment.

A wide variety of tools are available for capturing and analyzing I/O access from individual parallel applications, including IPM, HPCT-IO, LANL-Trace, IOT, and mpiP [20], [21], [22], [23], [24]. Multiple I/O tracing mechanisms were surveyed by Konwinski et al. [25]. Klundt, Weston, and Ward have also investigated tracing of user-level I/O libraries on lightweight kernels [26].

\section{CONCLUSIONS}

In this work we have introduced a methodology for continuous, scalable, production characterization of I/O workloads, and we have demonstrated its value in both understanding system behavior and accelerating debugging of individual applications. We used data collected over a two-month period to investigate critical questions about the nature of storage access characteristics on leadership-class machines. We performed our investigation on Intrepid, a 557-teraflop IBM Blue 
Gene/P deployed at Argonne National Laboratory. Intrepid's storage system contained over 191 million files and moved an average of nearly $150 \mathrm{TiB}$ of data per day. We captured detailed application-level I/O characteristics of $27 \%$ of all jobs executed on Intrepid, ranging in size from 1 to 163,840 processes. In doing so, we demonstrated that it is possible to instrument several aspects of storage systems at full scale without interfering with production users. We have also developed a performance metric that enables relative comparison of a wide variety of production applications.

The findings of this study will influence future research directions as well as the design of future $\mathrm{I} / \mathrm{O}$ subsystems to be used at the ALCF. We found that POSIX is still heavily used by many applications, although on the BG/P it offered no discernible performance advantage. MPI-IO, HDF5, and Parallel NetCDF are also used by the top $10 \mathrm{I} / \mathrm{O}$ producers and consumers. We also found several examples of I/O performance being constrained by the metadata overhead that results from accessing large numbers of small files. These examples of suboptimal behavior highlight the need for tools such as Darshan to simplify the task of understanding and tuning I/O behavior.

Shared or partially shared file usage becomes the predominant method of file access at the 16,384-processor mark on Intrepid. This job size, and larger, will be increasingly common on the next generation of computing platforms. This implies that the ALCF should invest in helping applications transition to shared or partially shared file models. We demonstrated the benefit of this approach through a case study of I/O tuning in the CombustionPhysics project, one of the most active INCITE projects on Intrepid in terms of data usage.

From the aspect of I/O system design, we found two major items of interest. We were able to verify the "burstiness" of $\mathrm{I} / \mathrm{O}$ on Intrepid, indicating that we have a significant opportunity to utilize idle storage resources for tasks such as performance diagnosis. In addition, we found that files are rarely overwritten once they are closed. This suggests that there is an opportunity to leverage hierarchical storage for more cost-effective storage of infrequently accessed data. We also found that while $\mathrm{I} / \mathrm{O}$ characterization data was easy to use on a per job basis, analyzing and summarizing many jobs in aggregate were more difficult than anticipated. As a result, we have enhanced Darshan in the 2.0.0 release to streamline the analysis process [27]. For example, more shared file statistics (such as minimum, maximum, and variance among participating processes) are now computed at run time.

We compared storage access characteristics of different scientific application domains and found an extraordinary variety of both data usage and application-level I/O performance. Several distinct I/O strategies were identified, including shared file usage, unique file usage, rank $0 \mathrm{I} / \mathrm{O}$, and examples of both weak and strong scaling of data. We also discovered examples of applications that appeared to be utilizing custom aggregation algorithms without the assistance of MPI-IO. In general we found that metadata overhead, small access sizes, and I/O imbalance were the most significant barriers to
I/O performance. However, no single technique employed by applications emerged overwhelmingly as the most successful. In future work we would like to perform similar studies at other sites that may offer a different collection of scientific computing applications to compare and contrast.

\section{ACKNOWLEDGMENTS}

This work was supported by Office of Advanced Scientific Computing Research, Office of Science, U.S. Dept. of Energy, under Contract DE-AC02-06CH11357.

This research used resources of the Argonne Leadership Computing Facility at Argonne National Laboratory, which is supported by the Office of Science of the U.S. Department of Energy under contract DE-AC02-06CH11357.

\section{REFERENCES}

[1] S. Lang, P. Carns, R. Latham, R. Ross, K. Harms, and W. Allcock, "I/O performance challenges at leadership scale," in SC '09: Proceedings of the Conference on High Performance Computing Networking, Storage and Analysis. New York, NY, USA: ACM, 2009, pp. 1-12.

[2] G. R. Ganger, "Generating representative synthetic workloads: An unsolved problem," in Proceedings of the Computer Measurement Group (CMG) Conference, 1995, pp. 1263-1269.

[3] A. Traeger, E. Zadok, N. Joukov, and C. P. Wright, "A nine year study of file system and storage benchmarking," Trans. Storage, vol. 4, no. 2, pp. 1-56, 2008.

[4] N. Agrawal, A. C. Arpaci-Dusseau, and R. H. Arpaci-Dusseau, "Towards realistic file-system benchmarks with CodeMRI," SIGMETRICS Perform. Eval. Rev., vol. 36, no. 2, pp. 52-57, 2008.

[5] E. Anderson, "Capture, conversion, and analysis of an intense NFS workload," in FAST '09: Proccedings of the 7th conference on File and storage technologies. Berkeley, CA, USA: USENIX Association, 2009, pp. $139-152$.

[6] A. W. Leung, S. Pasupathy, G. Goodson, and E. L. Miller, "Measurement and analysis of large-scale network file system workloads," in Proceedings of the 2008 USENIX Technical Conference. Berkeley, CA, USA: USENIX Association, 2008, pp. 213-226. [Online]. Available: http://portal.acm.org/citation.cfm?id=1404014.1404030

[7] P. Carns, R. Latham, R. Ross, K. Iskra, S. Lang, and K. Riley, "24/7 characterization of petascale I/O workloads," in Proceedings of 2009 Workshop on Interfaces and Architectures for Scientific Data Storage, September 2009.

[8] "U.S. Department of Energy INCITE program," http://www.er.doe.gov/ascr/incite/.

[9] F. Schmuck and R. Haskin, "GPFS: A shared-disk file system for large computing clusters," in Proceedings of the FAST 2002 Conference on File and Storage Technologies, January 2002.

[10] S. Godard, "SYSSTAT utilities home page," http://pagespersoorange.fr/sebastien.godard/.

[11] S. Dayal, "Characterizing HEC storage systems at rest," Carnegie Mellon University Parallel Data Lab, Tech. Rep. CMU-PDL-08-109, 2008.

[12] W. Liao and A. Choudhary, "Dynamically adapting file domain partitioning methods for collective I/O based on underlying parallel file system locking protocols," in Proceedings of the 2008 ACM/IEEE conference on Supercomputing. IEEE Press, Piscataway, NJ, 2008.

[13] N. Nieuwejaar, D. Kotz, A. Purakayastha, C. S. Ellis, and M. Best, "File-access characteristics of parallel scientific workloads," IEEE Transactions on Parallel and Distributed Systems, vol. 7, no. 10, pp. 1075-1089, October 1996. [Online]. Available: http://www.computer.org/tpds/td1996/11075abs.htm.

[14] Y. Kim, R. Gunasekaran, G. Shipman, D. Dillow, Z. Zhang, and B. Settlemyer, "Workload characterization of a leadership class storage cluster," in 5th Petascale Data Storage Workshop (PDSW), 2010, pp. 1 -5 .

[15] H. Yu, R. K. Sahoo, C. Howson, G. Almasi, J. G. Castanos, M. Gupta, J. E. Moreira, J. J. Parker, T. E. Engelsiepen, R. Ross, R. Thakur, R. Latham, and W. D. Gropp, "High performance file I/O for the BlueGene/L supercomputer," in Proceedings of the 12th International Symposium on High-Performance Computer Architecture, 2006. 
[16] E. Smirni and D. Reed, "Workload characterization of input/output intensive parallel applications," in Proceedings of the Conference on Modelling Techniques and Tools for Computer Performance Evaluation, ser. Lecture Notes in Computer Science, vol. 1245. Springer-Verlag, June 1997, pp. 169-180. [Online]. Available: http://vibes.cs.uiuc.edu/Publications/Papers/Tools97.ps.gz

[17] D. A. Reed, R. A. Aydt, R. J. Noe, P. C. Roth, K. A. Shields, B. W. Schwartz, and L. F. Tavera, "Scalable performance analysis: The Pablo performance analysis environment," in Proceedings of the Scalable parallel libraries conference. IEEE Computer Society, 1993, pp. 104113.

[18] F. Wang, Q. Xin, B. Hong, S. A. Brandt, E. L. Miller, D. D. E. Long, and T. T. Mclarty, "File system workload analysis for large scale scientific computing applications," in Proceedings of the 21st IEEE / 12th NASA Goddard Conference on Mass Storage Systems and Technologies, 2004, pp. $139-152$.

[19] A. Uselton, M. Hawison, N. Wright, D. Skinner, J. Shalf, L. Oliker, N. Keen, and K. Karavanic, "Parallel I/O performance: From events to ensembles," in Proceedings of the 24th IEEE International Parallel and Distributed Processing Symposium, 2010.

[20] N. J. Wright, W. Pfeiffer, and A. Snavely, "Characterizing parallel scaling of scientific applications using IPM," in 10th LCI International Conference on High-Performance Clustered Computing, 2009.
[21] S. Seelam, I.-H. Chung, D.-Y. Hong, H.-F. Wen, and H. Yu, "Early experiences in application level I/O tracing on Blue Gene systems," in Proceedings of the 2008 IEEE International Parallel and Distributed Processing Symposium, 2008.

[22] "HPC-5 open source software projects: LANL-Trace," http://institute.lanl.gov/data/software/\#lanl-trace.

[23] P. C. Roth, "Characterizing the I/O behavior of scientific applications on the Cray XT," in PDSW '07: Proceedings of the 2nd International Workshop on Petascale Data Storage. New York, NY, USA: ACM, 2007, pp. 50-55.

[24] J. S. Vetter and M. O. McCracken, "Statistical scalability analysis of communication operations in distributed applications," SIGPLAN Notices, vol. 36, no. 7, pp. 123-132, 2001.

[25] A. Konwinski, J. Bent, J. Nunez, and M. Quist, "Towards an I/O tracing framework taxonomy," in PDSW '07: Proceedings of the 2nd international workshop on Petascale data storage. New York, NY USA: ACM, 2007, pp. 56-62.

[26] R. Klundt, M. Weston, and L. Ward, "I/O tracing on Catamount," Sandia National Laboratory, Tech. Rep. SAND2008-3684, 2008.

[27] "Darshan," http://www.mcs.anl.gov/research/projects/darshan/. 